\title{
Technogenic Ecological Sequences in Tailing Pond from Căpușu Mare, Built between 1975-1981
}

\author{
Aurel MAXIM ${ }^{1}{ }^{*}$, Mignon ȘANDOR ${ }^{1}$, Adriana OPINCARIU ${ }^{1}$, Lucia MIHALESCU ${ }^{2}$, Vasile ROMAN ${ }^{1}$, Larisa BÎLC ${ }^{1}$ \\ ${ }^{1}$ University of Agricultural Sciences and Veterinary Medicine, Cluj-Napoca, Faculty of Agriculture, Calea \\ Mănăştur Street, 400372, Cluj-Napoca, Romania \\ ${ }^{2}$ Universitatea Tehnica din Cluj-Napoca, Centrul Universitar Nord Baia Mare, no.76, Victoriei Street, Baia \\ Mare, Roamnia \\ *corresponding author: aurel.maxim@usamvcluj.ro
}

Bulletin USAMV series Agriculture 74(2)/2017

Print ISSN 1843-5246; Electronic ISSN 1843-5386

DOI 10.15835/buasvmcn-agr: 0033

\begin{abstract}
In Romania, all actions related to mining management are found in "Strategy of the mining industry for 20122035". Today, numerous tailing ponds have remained outside rehabilitation operations, and they are becoming more natural, as is the case of the tailing pond in Capusu Mare. The vegetation study was conducted in 2015, at the tailing pond III in Căpușu Mare that was operational between 1975-1981. The following phytopopulation and phytocenotic indices were calculated: presence, frequency, class of presence, abundance-dominance and average abundance-dominance (ADm) of species. Floral studies show the presence of 40 plant species. The wood species with the highest mean dominant abundance are Hippophäe rhamnoides (14.78\%) and Salix alba (10.55\%), and of herbaceous species stands Phragmites communis with 7.49\%. After 34 years from the heap closure, the degree of vegetation coverage is $77 \%$. Wood species occupy about $32 \%$ of the heap surface.
\end{abstract}

Keywords: tailing pond, vegetation study, natural revegetation, phytopopulation and phytocenotic indices

\section{INTRODUCTION}

The European Union attaches great importance to reduce the impact of mining tailings ponds on the environment. The main Directive developed in this area is Directive 2006/21/EC of the European Parliament and of the Council of 15 March 2006 on the management of waste from extractive industries and amending Directive 2004/35/EC (Directive 2006/21/EC).

Programmatic document elaborated by the Government and mining industry entitled Strategy of the mining industry for the period 2012-2035 shows that, under the Mining Law 85/2003 was issued 11 government decisions which approved the closure of 556 mines/quarries. Since 1998 and until the end of 2011, 192 objectives have been closed and rehabilitated, at which we can add 53, with partially completed works (Strategia industriei miniere pentru perioada 2012-2035).
This process proves to be a difficult and costly one, given that Romania was a country subject to forced and aggressive industrialization until 1990. Thus, many tailing ponds have remained outside rehabilitation operations, and they have entered into a process of natural grassing. In this situation, there is also the tailings pond in Căpuşu Mare, which is the object of our study.

Under this circumstances, the cover with spontaneous vegetation of tailing ponds is ecologically beneficial as wind erosion and the transport of dust and toxic materials in the river basin to which they are part or neighbouring are reduced. Vegetation also brings with it aesthetic advantages (Maxim, 2008).

In Căpușu Mare, Cluj county, iron ore was exploited, resulting in four tailings dumps completed in 1969 (keep I), 1975 (keep II), 1981 (keep III) and 1985 (keep IV) (Clapa, 2003; Mâț, 
2012; Maxim et. al., 2015). This paper presents a vegetation study conducted in 2015 at the tailings heap III built between 1975 and 1981 (Fig. 1).

\section{MATERIALS AND METHODS}

Floral description was made using botanical determinants. The determination of phytopathogenic indices (presence, abundance-dominance, frequency) was done by the geobotanic method. For establishing the abundance-dominance indicator, the 7-grade Braun-Blanquet scale was used, as described by Maxim et al. (2015). Subsequently, the raw table was compiled, and on the basis of these data, each species ran into a class of presence (1-20, 20-40, 40-60, 61-80, 81-100\%), then the abundance- the average dominance was calculated (Maxim et al., 2016; Păcurar and Rotar, 2014). In addition to the specific coverage, the general cover of the dump with vegetation was also appreciated.

Soil analysis shows that the $\mathrm{pH}$ of the soil in vegetation-covered areas is 5.4 and in uncove- red portions of vegetation is 4.9. There is no data on the natural attenuation of the pollution phenomenon, but naturally it is assumed that the concentrations of the pollutants have decreased by self-purification.

\section{RESULTS AND DISCUSSION}

34 years after the tailings pond closure, vegetation coverage is $77 \%$, and wood species occupy approximately $32 \%$ of the landfill area. The floral inventory shows the presence of 40 species, as follows: 8 species of trees, 3 species of shrubs and 29 grass species.

The tree species are as follows: Betula pendula, Salix alba, Populus tremula, Populus alba, Pirus piraster, Salix fragilis, Robinia pseudoacacia and Acer negundo. It has been found the presence of three species of bushes, respectively, Hippophäe rhamnoides, Rubus fructicocus and Salix caprea.

The grass vegetation on the waste dumps is represented by 29 species: Phragmites communis, Festuca sp., Calamagrostis arundinacea subsp.

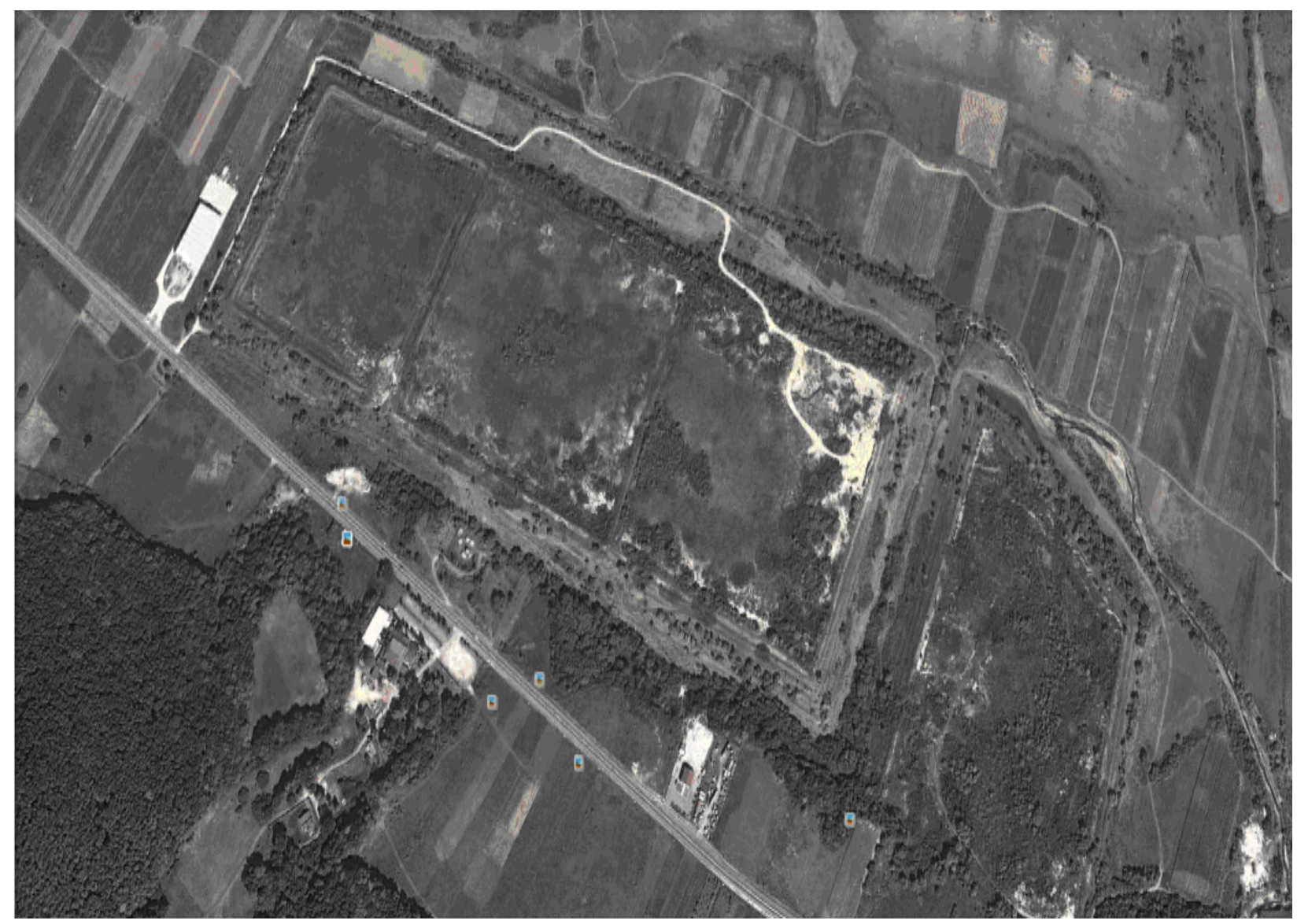

Fig. 1. The tailing ponds from Căpuşu Mare, Cluj - a satellite image (www.google.com) 
Tab. 1. The presence of identified vegetable species on the TAILING DEPOSIT III (1975-1981) from Căpușu Mare, Cluj County, in the year 2015

Species

Survey no./abundance-dominance

Pre-

sence

\begin{tabular}{|c|c|c|c|c|c|c|c|c|c|c|c|c|c|c|c|c|c|c|c|c|c|}
\hline & 1 & 2 & 3 & 4 & 5 & 6 & 7 & 8 & 9 & 10 & 11 & 12 & 13 & 14 & 15 & 16 & 17 & 18 & 19 & 20 & \\
\hline \multicolumn{22}{|c|}{ Trees } \\
\hline Salix alba & - & - & - & - & 1 & 2 & 1 & - & - & 2 & - & - & 2 & - & 3 & 3 & 2 & 2 & - & 3 & 10 \\
\hline Populus tremula & r & - & - & 3 & - & - & - & $\mathrm{r}$ & 1 & - & r & - & + & - & - & + & - & - & 1 & - & 8 \\
\hline Betula pendula & - & - & - & - & $\mathrm{r}$ & - & - & 1 & - & + & 1 & - & - & - & 2 & 2 & - & - & - & 2 & 7 \\
\hline Acer negundo & - & - & - & - & - & 2 & - & - & - & 2 & - & 2 & - & - & - & - & 1 & 2 & - & - & 5 \\
\hline Salix fragilis & - & - & - & - & 2 & 2 & - & - & - & - & - & - & 1 & 1 & 1 & - & - & - & - & - & 5 \\
\hline Populus alba & - & - & - & - & 2 & - & - & - & 3 & - & - & - & - & 2 & - & - & - & - & 1 & - & 4 \\
\hline Pirus piraster & - & - & - & - & - & - & 1 & - & - & - & - & 2 & - & - & - & $\mathrm{r}$ & - & - & - & - & 3 \\
\hline $\begin{array}{l}\text { Robinia } \\
\text { pseudoacacia }\end{array}$ & 4 & - & 3 & - & - & 3 & - & - & - & - & - & - & - & - & - & - & - & - & - & - & 3 \\
\hline
\end{tabular}

\section{Shrubs}

\begin{tabular}{|c|c|c|c|c|c|c|c|c|c|c|c|c|c|c|c|c|c|c|c|c|c|}
\hline $\begin{array}{l}\text { Hippophaё } \\
\text { rhamnoides }\end{array}$ & 1 & - & - & - & - & 3 & - & - & - & 3 & 2 & 2 & - & 2 & - & 4 & - & 3 & - & 4 & 9 \\
\hline Rubus fructicosus & - & - & 1 & 1 & - & - & - & 1 & 2 & - & - & - & - & - & 1 & - & - & - & - & 2 & 6 \\
\hline Salix caprea & 1 & - & - & - & $\mathrm{r}$ & - & - & - & - & - & - & - & - & - & - & - & - & - & - & - & 2 \\
\hline \multicolumn{22}{|c|}{ Herbaceous plants } \\
\hline $\begin{array}{l}\text { Phragmites } \\
\text { communis }\end{array}$ & - & 1 & 2 & 1 & + & - & 2 & 2 & + & + & 2 & 1 & 3 & + & - & - & 2 & + & 1 & - & 15 \\
\hline $\begin{array}{l}\text { Calamagrostis } \\
\text { arundinacea } \\
\text { subsp. Brachytri- } \\
\text { cha (Steud.) }\end{array}$ & - & - & $\mathrm{r}$ & $\mathrm{r}$ & - & 2 & - & 2 & - & - & 1 & - & 1 & + & + & - & 1 & + & - & - & 10 \\
\hline Festuca sp. & - & - & $\mathrm{r}$ & - & 1 & 1 & 2 & 2 & - & - & + & 1 & & - & + & $\mathrm{r}$ & - & - & - & - & 9 \\
\hline Vicia sativa & - & - & - & - & - & - & - & 1 & 1 & - & + & 1 & 2 & - & - & 2 & - & + & - & + & 8 \\
\hline Dactylis glomerata & - & - & 2 & 1 & - & + & - & $\mathrm{r}$ & - & - & 1 & - & $\mathrm{r}$ & - & + & - & + & - & - & - & 8 \\
\hline Poa nemoralis & - & - & $\mathrm{r}$ & + & + & $\mathrm{r}$ & - & $\mathrm{r}$ & - & - & - & - & + & - & - & - & - & + & - & - & 7 \\
\hline $\begin{array}{l}\text { Galium album } \\
\text { (mollugo) }\end{array}$ & - & - & + & + & + & + & - & - & + & - & - & - & - & - & + & - & + & - & - & - & 7 \\
\hline $\begin{array}{l}\text { Trifolium } \\
\text { pratense }\end{array}$ & - & - & - & + & - & - & 1 & 1 & - & - & 1 & + & - & - & + & - & - & 1 & - & - & 7 \\
\hline $\begin{array}{l}\text { Trifolium } \\
\text { montanum }\end{array}$ & - & - & - & - & - & - & $r$ & - & - & 1 & - & - & - & + & - & 1 & - & - & + & + & 6 \\
\hline Cichorium intybus & - & - & - & - & - & - & $\mathrm{r}$ & 1 & - & - & - & 1 & - & + & - & - & - & 1 & 1 & - & 6 \\
\hline Tussilago farfara & - & - & $\mathrm{r}$ & - & + & - & - & - & $\mathrm{r}$ & - & - & 1 & - & - & - & - & - & 1 & $\mathrm{r}$ & - & 6 \\
\hline $\begin{array}{l}\text { Hypericum } \\
\text { perforatum }\end{array}$ & - & - & - & - & + & 1 & - & 1 & - & - & 1 & - & - & - & + & - & - & + & - & - & 6 \\
\hline $\begin{array}{l}\text { Achillea } \\
\text { milefolium }\end{array}$ & - & - & - & - & + & + & - & $r$ & - & - & $\mathrm{r}$ & $\mathrm{r}$ & + & - & - & - & - & - & - & - & 6 \\
\hline $\begin{array}{l}\text { Onobrychis } \\
\text { viciifolia }\end{array}$ & - & - & - & $r$ & - & $r$ & - & - & - & - & $r$ & $r$ & - & - & $r$ & - & - & - & - & + & 6 \\
\hline Lotus corniculatus & + & - & - & 1 & $\mathrm{r}$ & - & 1 & - & - & - & + & - & - & - & - & - & $\mathrm{r}$ & - & - & - & 6 \\
\hline Sonchus arvensis & $\mathrm{r}$ & + & + & - & - & 1 & 1 & - & - & - & - & - & - & - & - & - & - & - & - & - & 5 \\
\hline
\end{tabular}




\begin{tabular}{|c|c|c|c|c|c|c|c|c|c|c|c|c|c|c|c|c|c|c|c|c|c|}
\hline \multirow{2}{*}{ Species } & \multicolumn{20}{|c|}{ Survey no./abundance-dominance } & \multirow{2}{*}{$\begin{array}{l}\text { Pre- } \\
\text { sence }\end{array}$} \\
\hline & 1 & 2 & 3 & 4 & 5 & 6 & 7 & 8 & 9 & 10 & 11 & 12 & 13 & 14 & 15 & 16 & 17 & 18 & 19 & 20 & \\
\hline $\begin{array}{l}\text { Plantago } \\
\text { lanceolata }\end{array}$ & - & - & + & - & - & + & - & - & - & - & + & - & + & - & - & - & + & - & - & - & 5 \\
\hline Daucus carota & - & - & + & - & $\mathrm{r}$ & - & - & $\mathrm{r}$ & $\mathrm{r}$ & - & - & - & - & - & - & - & - & - & $r$ & - & 5 \\
\hline Cirsium arvense & $\mathrm{r}$ & r & - & - & - & - & - & - & - & - & + & - & - & - & $\mathrm{r}$ & - & + & - & - & - & 5 \\
\hline Kanutia arvensis & - & - & - & - & - & - & - & - & - & - & 1 & - & + & - & - & 1 & - & - & + & + & 5 \\
\hline $\begin{array}{l}\text { Leuchantemum } \\
\text { vulgare }\end{array}$ & - & + & - & $\mathrm{r}$ & - & - & + & 1 & - & - & $r$ & - & - & - & - & - & - & - & - & - & 5 \\
\hline Carex sp. & - & - & - & - & $\mathrm{r}$ & - & - & + & - & - & + & - & - & - & + & - & - & - & - & - & 4 \\
\hline Vicia craca & - & - & - & - & + & $\mathrm{r}$ & - & - & - & - & - & - & - & - & - & - & + & - & + & - & 4 \\
\hline Medicago sativa & - & - & - & - & 1 & - & - & - & - & + & - & + & - & - & - & - & - & - & + & - & 4 \\
\hline $\begin{array}{l}\text { Equisetum } \\
\text { arvense }\end{array}$ & 1 & - & - & $\mathrm{r}$ & - & - & - & + & - & - & - & - & - & - & - & - & - & - & - & - & 3 \\
\hline $\begin{array}{l}\text { Rhianthus } \\
\text { angustifolius }\end{array}$ & - & - & - & - & - & - & r & - & + & - & - & - & - & - & - & - & - & - & + & - & 3 \\
\hline Papaver rhoeas & $\mathrm{r}$ & - & - & - & - & - & - & - & - & - & - & - & $r$ & - & - & - & - & - & - & - & 2 \\
\hline
\end{tabular}

Brachytricha (Steud.), Galium album (mollugo), Tussilago farfara, Vicia sativa, Cirsium arvense, Poa nemoralis, Hypericum perforatum, Trifolium pratense, Trifolium montanum, Cichorium intybus, Dactylis glomerata, Onobrychis viciifolia, Medicago sativa, Achillea milefolium, Lotus corniculatus, Plantago lanceolata, Leuchantemum vulgare, Kanutia arvensis, Daucus carota, Sonchus arvensis, Vicia craca, Stipa pennata, Carex sp., Silene vulgaris, Rhianthus angustifolius, Equisetum arvense and Papaver rhoeas.

The presence of trees varies between 3 and 10 markers, out of the 20. At shrubs the presence is 9 at Hippophäe rhamnoides and 2 at Salix caprea. In herbaceous plants, the values of this indicator range between much larger values, from 15 at Phragmites communis up to 2 at Papaver rhoeas.

In Table 1, the species in the raw table were transcribed in order of their descending order. In trees, Salix alba (P = 10), Populus tremula (8), Betula pendula (7), Acer negundo (5), Salix fragilis (5), Populus alba (4), Pirus piraster (3), Robinia pseudoacacia (3), and in shrubs, Hippophaë rhamnoides (9), Rubus fructicosus (6) and Salix caprea (2). As for the grass species, the presence in descending order was as follows: Phragmites communis (15), Calamagrostis arundinacea subsp. Brachytricha (Steud.) (10), Festuca sp. (9), Vicia sativa (8), Dactylis glomerata (8), Poa nemoralis (7), Galium album (mollugo) (7), Trifolium pratense (7), Trifolium montanum (6), Cichorium intybus
(6), Tussilago farfara (6), Hypericum perforatum (6), Achillea milefolium (6), Onobrychis viciifolia (6), Lotus corniculatus (6), Sonchus arvensis (5), Plantago lanceolata (5), Daucus carota (5), Cirsium arvense (5), Kanutia arvensis (5), Leuchantemum vulgare (5), Carex sp. (4), Vicia craca (4), Medicago sativa (4), Equisetum arvense (3), Silene vulgaris (3), Stipa pennata (3), Rhianthus angustifolius (3) and Papaver rhoeas (2).

The frequency of the species and the 5 classes of presence are transposed in Table 2 .

On trees, the most widespread species was Salix alba, with a frequency of $50 \%$, followed bye Populus tremula with $40 \%$, Betula pendula with $35 \%$, Salix fragilis and Acer negundo with 25\%, Populus alba with 20\%, Pirus piraster and Robinia pseudoacacia with $15 \%$. One species falls under Class III of presence (Salix alba), four under Class II (Populus tremula, Betula pendula, Salix fragilis, Acer negundo), and the remaining three (Pirus piraster, Robinia pseudoacacia and Populus alba), in Class I of presence.

Shrubs are found in $45 \%$ of the surveys at Hippophäe rhamnoides, $30 \%$ at Rubus fructicocus, and $10 \%$ at Salix caprea and in terms of class of presence, Hippophäe falls under Class III, Rubus fructicocus falls within Class II and Salix caprea falls within Class I.

The most widespread herbaceous species on the tailings dumps at Căpuşu Mare, Phragmites communis, has a frequency of $75 \%$ of the surveys, 
Tab. 2. Frequency, class presence and average abundance-dominance of vegetable species on the TAILING DEPOSITI III (1975-1981), from Căpușu Mare, Cluj County, in the year 2015

\begin{tabular}{|c|c|c|c|}
\hline Species & Frequency (\%) & Class of presence (c.) & $\mathrm{AD}_{\mathrm{m}}$ \\
\hline \multicolumn{4}{|l|}{ Trees } \\
\hline Salix alba & 50 & III & $10.55 \%$ \\
\hline Populus tremula & 40 & II & $2.49 \%$ \\
\hline Betula pendula & 35 & II & $3.21 \%$ \\
\hline Acer negundo & 25 & II & $3.78 \%$ \\
\hline Salix fragilis & 25 & II & $2.58 \%$ \\
\hline Populus alba & 20 & $\mathrm{I}$ & $3.9 \%$ \\
\hline Pirus piraster & 15 & I & $1.15 \%$ \\
\hline Robinia pseudoacacia & 15 & I & $6.88 \%$ \\
\hline \multicolumn{4}{|l|}{ Shrubs } \\
\hline Hippophaë rhamnoides & 45 & III & $14.78 \%$ \\
\hline Rubus fructicosus & 30 & II & $2.85 \%$ \\
\hline Salix caprea & 10 & I & $0.28 \%$ \\
\hline \multicolumn{4}{|l|}{ Herbaceous plants } \\
\hline Phragmites communis & 75 & IV & $7.49 \%$ \\
\hline Calamagrostis arundinacea subsp. Brachytricha (Steud.) & 50 & III & $2.66 \%$ \\
\hline Festuca sp. & 45 & III & $2.64 \%$ \\
\hline Vicia sativa & 40 & II & $2.65 \%$ \\
\hline Dactylis glomerata & 40 & II & $1.51 \%$ \\
\hline Poa nemoralis & 35 & II & $0.12 \%$ \\
\hline Galium album (mollugo) & 35 & II & $0.19 \%$ \\
\hline Trifolium pratense & 35 & II & $1.18 \%$ \\
\hline Trifolium montanum & 30 & II & $0.64 \%$ \\
\hline Cichorium intybus & 30 & II & $1.13 \%$ \\
\hline Tussilago farfara & 30 & II & $0.59 \%$ \\
\hline Hypericum perforatum & 30 & II & $0.91 \%$ \\
\hline Achillea milefolium & 30 & II & $0.09 \%$ \\
\hline Onobrychis viciifolia & 30 & II & $0.04 \%$ \\
\hline Lotus corniculatus & 30 & II & $0.61 \%$ \\
\hline Sonchus arvensis & 25 & II & $0.60 \%$ \\
\hline Plantago lanceolata & 25 & II & $0.13 \%$ \\
\hline Daucus carota & 25 & II & $0.04 \%$ \\
\hline Cirsium arvense & 25 & II & $0.06 \%$ \\
\hline Kanutia arvensis & 25 & II & $0.63 \%$ \\
\hline Leuchantemum vulgare & 25 & II & $0.33 \%$ \\
\hline Carex sp. & 20 & I & $0.09 \%$ \\
\hline Vicia craca & 20 & I & $0.09 \%$ \\
\hline Medicago sativa & 20 & I & $0.36 \%$ \\
\hline Equisetum arvense & 15 & I & $0.31 \%$ \\
\hline Silene vulgaris & 15 & $\mathrm{I}$ & $0.008 \%$ \\
\hline Stipa pennata & 15 & I & $0.58 \%$ \\
\hline Rhianthus angustifolius & 15 & I & $0.06 \%$ \\
\hline Papaver rhoeas & 10 & I & $0.055 \%$ \\
\hline
\end{tabular}


followed by Calamagrostis arundinacea subsp. Brachytricha (Steud.) with 50\%, Festuca sp. with $45 \%$, Vicia sativa with $40 \%$,

Dactylis glomerata with 40\%, Poa nemoralis with 35\%, Galium album (mollugo) 3 with 5\%, Trifolium pratense with 35\%, Trifolium montanum with 30\%, Cichorium intybus with 30\%, Tussilago farfara with 30\%, Hypericum perforatum with 30\%, Achillea milefolium with 30\%, Onobrychis viciifolia with $30 \%$, Lotus corniculatus with 30\%, Sonchus arvensis with 25\%, Plantago lanceolata with 25\%, Daucus carota with 25\%, Cirsium arvense with $25 \%$, Kanutia arvensis with 25\%, Leuchantemum vulgare with $25 \%$, Carex sp. with $20 \%$,Vicia craca with $20 \%$, Medicago sativa with $20 \%$, Equisetum arvense with 15\%, Silene vulgaris with 15\%, Stipa pennata with 15\%, Rhianthus angustifolius with 15\% and Papaver rhoeas with 10\%.

As far as the classes of presence are concerned, of the 29 herbaceous species, one belongs to the fourth class (Phragmites communis), two fall in class III (Calamagrostis arundinacea subsp. Brachytricha (Steud.) and Festuca sp.), 18 in class II (Vicia sativa, Dactylis glomerata, Poa nemoralis, Galium album (mollugo), Trifolium pratense, Trifolium montanum, Cichorium intybus, Tussilago farfara, Hypericum perforatum, Achillea milefolium, Onobrychis viciifolia, Lotus corniculatus, Sonchus arvensis, Plantago lanceolata, Daucus carota, Cirsium arvense, Kanutia arvensis, Leuchantemum vulgare), eight in Class I (Carex sp., Vicia craca, Medicago sativa, Equisetum arvense, Silene vulgaris, Stipa pennata, Rhianthus angustifolius, Papaver rhoeas).

The mean abundance-dominance indicates the average coverage of a species of all the achieved results. On Hump III in Căpușu Mare, this indicator recorded values higher than 5 for the following species: Hippophäe rhamnoides (14.78\%), Salix alba $(10.55 \%)$ and Phragmites communis cu $7.49 \%$.

The plant species present on the tailing ponds are also found around these ponds, except Hippophäe rhamnoides, which prefer acidic $\mathrm{pH}$ soils.

\section{CONCLUSION}

The tailings pond III from Căpuşu Mare operated for about 6 years, from 1975 until 1981.

After 34 years since the heap was closed, wood species occupied about $32 \%$ of the heap's surface, with the general vegetation coverage of $77 \%$. Floral studies show the presence of 40 species, of which 8 species of trees, 2 species of shrubs and 30 grass species.

The woody species with the highest mean abundance-dominance are Hippophäe rhamnoides $(14.78 \%)$ and Salix alba (10.55\%), and of herbaceous species stands Phragmites communis with $7.49 \%$.

\section{REFERENCES}

1. Clapa D (2003). Cercetări privind protosolurile antropice din Transilvania, Cluj-Napoca. Teză de doctorat, ClujNapoca.

2. Maxim A (2008). Ecologie generală și aplicată. Ed. Risoprint, Cluj-Napoca.

3. Maxim A, Stoie A, Șandor M, Odagiu A, Pertea V, Mihalescu $\mathrm{L}$ (2015). The Natural Rehabilitation of Tailing Ponds from Căpuș, Cluj. Bulletin of University of Agricultural Sciences and Veterinary Medicine Cluj-Napoca, Agriculture, 72 (1):154-160

4. Maxim A, Șandor M, Opincariu A, Perțea V, Mihalescu L, Roman V (2016). Technogenic Ecological Sequences in Mine Tailings from Căpușu Mare, Built between 19691975, ProEnvironment, 9: 435441.

5. Mâţ S (2012). Structura și dinamica vegetației pe haldele de steril de la Căpuş, Cionca și Aghireș (jud. Cluj), Teză de doctorat, Cluj- Napoca.

6. Păcurar F, Rotar I (2014). Metode de studiu și interpretare a vegetației pajiștilor. Editura Risoprint, Cluj-Napoca.

7. Directive 2006/21/ EC of the European Parliament and of the Council of 15 March 2006 on the management of waste from extractive industries and amending Directive 2004/35/EC - Statement by the European Parliament, the Council and the Commission.

8. Strategia industriei miniere pentru perioada 2012-2035. Ministerul Economiei.

9. www.google.com. 